\title{
Recurso instruccional para el desarrollo del contenido de estructuras mediante la aplicación de la realidad aumentada en el área de tecnología. caso: colegio "Pablo Correa León” Cúcuta, Colombia
}

\section{Instructional resource for the development of content structures through the application of augmented reality in the area of technology. case: school "Pablo Correa León" Cúcuta, Colombia}

\author{
Indira Johana Cacua-Peñaloza ${ }^{\mathrm{a}}$ \\ ${ }^{a}$ Magister en Innovaciones Educativas, indykqa@gmail.com, Orcid: 0000-0002-4368-9085. Universidad Pedagógica Experimental Libertador. \\ Cúcuta, Colombia
}

Forma de citar: Cacua-Peñaloza I.J. Recurso instruccional para el desarrollo del contenido de estructuras mediante la aplicación de la realidad aumentada en el área de tecnología. caso: colegio "Pablo Correa León" Cúcuta, Colombia. Eco Matemático, 9(1), 97-121

Recibido: Agosto 06 de 2017

Aceptado: Diciembre 20 de 2017

\begin{abstract}
Palabras clave
Recurso instruccional, estructuras, realidad aumentada
\end{abstract}

\begin{abstract}
Resumen: La presente investigación se desarrolló desde la perspectiva de: Proponer un recurso instruccional apoyado en la realidad aumentada para el desarrollo de los contenidos de estructuras del área de tecnología en los estudiantes de séptimo grado de la institución Educativa Colegio "Pablo Correa León", ubicada en el municipio de Cúcuta, para ello, se consideró pertinente desarrollar una investigación amparada en los postulados de la investigación cuantitativa, con énfasis en la modalidad proyecto factible, con un diseño de campo y un nivel descriptivo, se empleó como población a los estudiantes de referida institución educativa, para lo cual se diseñó el instrumento de recolección de la información, el cual arrojó datos que permitieron el análisis y la interpretación de los datos desde cada uno de los objetivos del estudio. En el diagnostico se logró establecer que los docentes emplean estrategias didácticas poco adecuadas en el desarrollo de los contenidos de estructuras, de igual manera, es necesario sostener que las herramientas y recursos que desde la tecnología promueven el empleo de la realidad aumentada, son significativas, aunque no son empleadas por los docentes de manera adecuada, de igual forma se logró establecer que existe una muy alta factibilidad de aplicación de un recurso instruccional apoyado en la realidad aumentada para el desarrollo de los contenidos de estructuras del área de tecnología en los estudiantes de séptimo grado de la institución Educativa Colegio "Pablo Correa León", ubicada en el municipio de Cúcuta, razón por la cual se generó el recurso instruccional.
\end{abstract}

* Autor para correspondencia: indykqa@gmail.com

http://dx.doi.org/10.22463/17948231.1732

2590-9215 C 2018 Universidad Francisco de Paula Santander. Este es un artículo bajo la licencia CCBY 


\section{Keywords}

Instructional resource, structures, augmented reality

\section{Introducción}

El desarrollo tecnológico impone la consecución de evidencias que sirven de base para comprender el desarrollo de tal situación dentro de la realidad, es así como la misma se hace presente incluso en los contextos escolares, donde la tecnología ha llegado a dinamizar el desarrollo de contenidos, pero además de ello, se hace presente en los estudiantes, dado que es un área más dentro de los currículos de formación escolar, en este sentido, el área de tecnología, es uno de los elementos que ponen en evidencia situaciones inherentes a la constitución de un pensamiento donde lo tecnológico cobre una real vigencia.

En este sentido, es necesario sostener que el área de tecnología agrupa dentro de sí, una serie de contenidos complejos, que en su totalidad pueden conformar conocimientos significativos, al respecto es necesario sostener que para establecer la efectividad de la investigación, es necesario centrarse en el abordaje de un contenido en específico, por ello, asumir el contenido de estructuras es fundamental para el desarrollo del presente estudio, en este sentido, es necesario formular un recurso instruccional que permita el desarrollo del contenido de estructuras mediante el uso de la realidad aumentada.

Por lo tanto, el contenido de estructuras hace referencia a la organización y orden de las diferentes parte de un objeto, lo cual debe corresponderse con la utilidad del mismo, de allí la necesidad de desarrollar un estudio amparado en los postulados del método científico, donde se haga énfasis en un proceso de producción del conocimiento que permita formular opciones didácticas que impacten en el desarrollo de contenidos del área de tecnología con énfasis en tendencias actuales 
que sirvan de base en el desarrollo de situaciones propias dentro de la realidad.

\section{Situación problemática del estudio}

Los múltiples cambios significativos originados gracias a las Tecnologías de Información y Comunicación (TIC), han generado un gran paradigma en los procesos educativos, que han llevado a la necesidad de actualizar, optimizar y profundizar los procesos de enseñanzay aprendizaje para mejorar la calidad de la educación y llevar a cabo un cambio en los procesos productivos y desarrollo de la sociedad actual.

Según Guerra y Jordán (2010). En América Latina las políticas públicas en relación a las TIC se iniciaron hacia mediados de los años noventa con la intención de los gobiernos de reducir la brecha digital y prestar gran atención de su aplicación en los procesos educativos. Ante este aspecto, fue necesario desarrollar una política pública con el fin de implementar "una economía mundial basada en el saber", con el objetivo de revisar los enfoques y las prácticas de uso y de evaluación del impacto de TIC en la calidad de la educación de América Latina y el Caribe. Además de la necesidad profundizar, innovar y flexibilizar las prácticas docentes basadas en la utilización de las TIC en miras de una educación más globalizada, rica en interculturalidad, apoyada en aprendizajes colaborativos y significativos dejando a un lado así los métodos tradicionales y adaptando la educación a procesos acorde a los tiempos, dispositivos $\mathrm{y}$ herramientas disponibles.

De ahí, la necesidad de los diferentes países de ejecutar distintos programas y proyectos de incorporación de las TIC en la educación, y Colombia no siendo ajena a estos cambios y con el fin de avanzar en las trasformaciones que la educación necesita, asume su compromiso con la elaboración del plan maestro, Plan Nacional Decenal de Educación (PNDE) (2006-2016), donde plasma dentro de sus propósitos y objetivos la incorporación de las TIC para el mejoramiento de la educación y propenda el desarrollo sostenible del país, como lo señala en su documento (2006):

En el 2016 se contará con estructuras curriculares flexibles y pertinentes articuladas al desarrollo de las capacidades de aprender a ser, aprender a aprender y aprender a hacer y de las dimensiones científicas, técnicas, tecnológicas, humanísticas y artísticas, y a las competencias en una segunda lengua en ambientes de aprendizaje, contextualizados e incluyentes, que privilegien el uso y la apropiación de las TIC. (p.5)

Por esta razón, el gobierno Colombiano en su renovación pedagógica desde y uso de las TIC en la educación, establece políticas para incorporar masivamente la adopción de procesos de enseñanza y aprendizaje que permitan el acceso a la información, al conocimiento, al uso de tecnologías actualizadas de información y comunicación, ampliando la cobertura de conectividad y acceso a las redes, además de dotar a las instituciones de recursoes que garanticen la interacción de los actores educativos, haciendo énfasis en la formación del educando.

Sin embargo, para llevar a cabo esta política se requiere de un recurso humano capaz de generar y usar el conocimiento para la trasformación del país, participando activamente en la la aplicación y desarrollo de herramientas, para el apoyo del proceso de enseñanza y aprendizaje basados en la integración de las TIC en el aula, por lo se requiere de maestros actualizados para enfrentar estos nuevos desafíos, evaluando su quehacer pedagógico e incorporando instrumentos que permitan potencializar habilidades cognitivas $\mathrm{y}$ actitudes que generen un aprendizaje significativo. Para llevar a cabo este proceso es necesario incluir nuevos métodos de enseñanza con el uso directo de los servicios TIC con el objeto de mejorar los niveles de rendimiento de los estudiantes, la articulación de saberes de forma eficiente, contextualizada y proyectada hacia la construcción de un conocimiento. 
La realidad del aula escolar permite a los maestros observar cómo los educandos, a pesar de recibir aparentemente los mismos contenidos y explicaciones, aprenden de forma distinta las temáticas abordadas, en investigaciones realizadas por Gardner (1983), citadas por De Luca (2000), concluye en su análisis sobre el docente y las inteligencias múltiples, que estas diferencias podrían relacionarse con distintas maneras de aprender. De ahí que existen diversos estilos de aprendizaje que se relacionan directamente con las estrategias individuales e inconscientes utilizadas por el educando para aprender algo. Por lo tanto es inherente la incorporación de las TIC como agente integrador de las múltiples formas, formatos y lenguajes con los que se desea brindar un aprendizaje que les permita acceder al conocimiento desde su diversidad.

Por lo anterior, considerando lo señalado es necesario replantear los procesos que se siguen en la Escuela para estimular la creatividad, la incorporación de TIC contribuye indudablemente a consolidar procesos significativos de aprendizaje inter y trasdisciplinar, conducentes a la generación y estructuración de un pensamiento divergente.

Lo anterior reafirma la necesidad de evaluar y dar múltiples soluciones e interpretaciones a una problemática, un contexto, en este caso particular, a un recurso escolar o una tecnología audiovisual, debido a que los docentes no informáticos, les cuesta mucho incorporar las nuevas tecnologías en sus clases, la aplicación nuevos de recursoes educativos, además de documentar sus aplicaciones, entre otros.

El documento Colombia en PISA 2012 (2013) del Ministerio de Educación Nacional, señala el bajo desempeño en las pruebas nacionales, e internacionales, como las Pruebas Saber y el Programa de Evaluación Internacional de Estudiantes (PISA) respectivamente, reflejando la necesidad de mejorar la educación de todos los estudiantes en Ciencias para que puedan comprender el mundo tecnológico en el que viven y participar activamente en él, por tanto, los nuevos formatos escolares basados en las TIC deben convertirse para el educando en una nueva forma de expresión y comunicación de contenidos curriculares a través de la imagen, el video, el texto virtual, entre otros.

El Fondo de las Naciones Unidas para la Infancia (UNICEF), resume en su documento las experiencias y políticas que buscan agregar valor a la educación colombiana con apoyo de TICE Tecnologías de Información y Comunicación en Educación, en miras a transformaciones sociales y económicas.

La aplicación de la tecnología en la enseñanza genera experiencias de innovación educativa permitiendo al estudiante como lo señala Del Moral (1999) "formarse una idea del mundo, de tal modo que primero aprehenda la realidad (observe, compare, imagine) y después la exprese (represente, construya), logrando así desarrollar la capacidad creativa, entendida como ideación y comunicación” (p. 46).

De la Torre, J. y otros (2011) señalan que para lograr una mejor comprensión y un mejor aprendizaje, las tecnologías gráficas influyen en la manera en que los usuarios pueden manipular la realidad, ofreciendo la posibilidad de sustituir los modelos físicos por modelos virtuales con los que pueda interactuar de forma intuitiva. Entre estas tecnologías destacan la realidad aumentada (RA) y los dispositivos multitáctiles. Por tanto la aplicación de herramientas basadas en estas tecnologías debe convertirse para el educando en una forma de expresión que le permita evaluar y dar múltiples soluciones e interpretaciones a una problemática, un contexto, en este caso particular, a un recurso escolar o una tecnología audiovisual.

Partiendo de los diferentes enfoques tecnológicos disponibles en el mercado de gran utilidad para los procesos de enseñanza y aprendizaje, y dentro de las múltiples alternativas como es el caso de la RA que según el Azuma 
(1997) "permite al usuario ver el mundo real, con objetos virtuales superpuestos en, o mezclados con el mundo real", lo que resultaría de gran utilidad para el desarrollo de contenido para los nuevos esquemas de aprendizaje para nuestros estudiantes, que quieren aprender de nuevas formas.

Sin embargo, la incorporación de la RA en el campo educativo Colombiano en diversas áreas apenas está en sus inicios, donde algunos estudios y aplicaciones han demostrado su utilidad. Monal (2012), propone la RA como una herramienta tecnológica, para apoyar la exploración de las problemáticas del ecosistema bosque seco tropical aledaño a la institución educativa ciudad de Cartago. Novoa, (2013) propone el desarrollo de competencias musicales mediante un dispositivo interactivo con realidad aumentada, entre otros trabajos que aplican esta herramienta TIC para involucrar a los estudiantes de forma activa $\mathrm{y}$ participativa en los procesos de enseñanza $y$ aprendizaje.

La institución educativa Pablo Correa León de Cúcuta, Colombia, no es ajena a la necesidad de implementar las TIC en sus procesos de formación, ya que en el proyecto educativo institucional PEI, propone la necesidad de implementar las TIC como recursos para fortalecer los procesos de enseñanza y aprendizaje e ir acorde a los cambios tecnológicos que la sociedad lo requiere. Generando nuevas oportunidades para la enseñanza y el aprendizaje, cambiando las prácticas educativas tradicionales, evaluando la profesión y la práctica docente, frente a una realidad que les exige incorporarse a un mundo tecnificado que les demanda conocimientos y destrezas de las que muchas veces carecen, hasta generar redes de aprendizaje que permitan la consolidación de prácticas pedagógicas libres de la rigidez que suponen los esquemas tradicionales de enseñanza y aprendizaje (Saurith, 2014)..

Dado que una de las falencias encontradas en las evaluaciones institucionales y en los reportes académicos, muestran la escasa integración de las TIC en el currículo y la implementación de metodologías tradicionales; siendo aún más notorio en el área de tecnología, donde se evidencia un bajo rendimiento, la falta de iniciativas del profesional docente en el uso, producción e implementación de recursos pertinentes que permitan orientar y generar en los estudiantes procesos de análisis y construcción de su propio conocimiento a partir de experiencias, incorporando tecnologías disponibles en el mercado de gran utilidad, haciendo uso de medios electrónicos que están al alcance de los estudiantes, quienes cada día demandan el uso de recursos tecnológicos atendiendo a los nuevos esquemas de aprendizaje.

Por lo tanto, la actual investigación se propone sentar bases para fortalecer el proceso de enseñanza y aprendizaje incorporando herramientas colaborativas e inspiradoras, para el caso de la institución Pablo Correa León específicamente los estudiantes de séptimo grado, en contexto para desarrollo de contenidos de estructuras, siendo este uno de los que presenta mayor dificultad y falta de interés en los estudiantes, con miras a dotar a los docentes, con una serie de elementos estratégicos que puedan ser incluidos dentro del proceso de enseñanza, con miras a lograr la misión de la educación.

Con la finalidad de lograr tal situación, es pertinente generar los siguientes interrogantes: ¿de qué manera proponer un recursoinstruccional apoyado en la realidad aumentada para el desarrollo de los contenidos de estructuras del área de tecnología en los estudiantes de séptimo grado de la institución Educativa Colegio "Pablo Correa León", ubicada en el municipio de Cúcuta?, ¿Cuáles son las estrategias didácticas empleadas por el docente para el desarrollo de los contenidos de estructuras?, ¿Cómo las herramientas y recursos que desde la tecnología promueven el empleo de la realidad aumentada?, ¿Cuál es la factibilidad de aplicación de un recursoinstruccional apoyado en la realidad aumentada para el desarrollo de los contenidos de estructuras del área de tecnología en los estudiantes de séptimo grado de la institución 
Educativa Colegio "Pablo Correa León”, ubicada en el municipio de Cúcuta?, ¿Cómo diseñar un recursoinstruccional para el desarrollo del contenido de estructuras mediante la aplicación de la realidad aumentada en el área de tecnología en la institución objeto de estudio?

\section{Objetivos del Estudio}

\section{Objetivo General}

Proponer un recursoinstruccional apoyado en la realidad aumentada para el desarrollo de los contenidos de estructuras del área de tecnología en los estudiantes de séptimo grado de la institución Educativa Colegio "Pablo Correa León", ubicada en el municipio de Cúcuta.

\section{Objetivos Específicos}

1. Diagnosticar las estrategias didácticas empleadas por el docente para el desarrollo de los contenidos de estructuras.

2. Describir las herramientas y recursos que desde la tecnología promueven el empleo de la realidad aumentada

3. Determinar la factibilidad de aplicación de un recurso instruccional apoyado en la realidad aumentada para el desarrollo de los contenidos de estructuras del área de tecnología en los estudiantes de séptimo grado de la institución Educativa Colegio "Pablo Correa León", ubicada en el municipio de Cúcuta

4. Diseñar un recurso instruccional para el desarrollo del contenido de estructuras mediante la aplicación de la realidad aumentada en el área de tecnología en la institución objeto de estudio.

\section{Justificación e Importancia del Estudio}

En vista de los contantes cambios a los cuales está sometida la sociedad, es necesario estar a la vanguardia de estas transformaciones y más aún en el campo de las TIC que han modificado drásticamente, la forma de trabajo, la manera de interactuar, de comunicar, al igual que, la forma como se accede al conocimiento y se aprende.

Los nuevos desafíos educativos requieren el compromiso de una educación de calidad, donde se demanda mejor cualificación docente, con el desarrollo de competencias y el perfeccionamiento de las prácticas pedagógicas acordes a los cambios que la sociedad lo requiere, mediante la innovación e incorporación de las TIC para el desarrollo de nuevas habilidades y saberes pertinentes a los procesos formativos.

El Ministerio de Educación Nacional (MEN) en su proceso de actualización y fomento hacía la innovación, establece pautas para docentes y directivos docentes para la integración de las TIC en las prácticas educativas con el desarrollo de competencias las cuales están definidas como: el conjunto de conocimientos, habilidades, actitudes, comprensiones y disposiciones cognitivas, socioafectivas y psicomotoras apropiadamente relacionadas entre sí para facilitar el desempeño flexible, eficaz y con sentido de una actividad en contextos relativamente nuevos y retadores (Ministerio de Educación Nacional, 2006).

Es así como la aplicación de las TIC para el desarrollo de competencias, permiten fortalecer las prácticas educativas respondiendo a las necesidades locales, regionales y nacionales, para lo cual la implementación de TIC en el contexto educativo, requiere de herramientas tecnológicas que se presten para fines pedagógicos como aparatos que despierten el interés de los estudiantes como el televisor, el proyector o el computador, también puede ser software con el que se pueda escribir, diseñar, graficar, animar o simular, además dispositivos que están aún más a su alcance como los celulares, cámaras o tabletas, con las que se pueden desarrollar una serie de estrategias didácticas, para lograr un aprendizaje significativo, dentro de los espacios escolares, especialmente en el área de tecnología, 
generando desde el entorno escolar, espacios con nuevas formas de acceder a la información y la posibilidad de proporcionar ricas experiencias de aprendizaje colaborativo mediante la interacción, con la posibilidad de acceder fácilmente.

Por tal razón, si se permite comprender desde de una mejor dimensión los conceptos de estructuras, desde una perspectiva completa, que impacte de manera positiva y significativa dentro de la realidad, donde el usuario pueda interactuar de una manera amigable con un objeto, como lo señala Novoa,(2013) a través de su razonamiento cognitivo simple es aquí donde se implementa el sentido usualmente más desarrollado por las personas: el visual, y que además es el más vinculado a todas las inteligencias múltiples y si dentro de esta interacción se disponen una serie de resultados lógicos a manera de método pedagógico constructivo, generara en el usuario un aprendizaje y finalmente un desarrollo de habilidades a partir de estímulos positivos como es el caso de la realidad aumentada, dado que esta tecnología permite la combinación real-virtual, interactividad, ubicuidad, mediante la proyección de imágenes, contenidos, sonidos, videos, facilitando al usuario percibir esta información en el mismo lugar en que se encuentra.

Según Reinoso (2014), en los últimos años, son muchos los estudios que han demostrado las posibilidades que ofrece esta tecnología en campos tan distintos como: la medicina, el turismo, en museos, educación, donde ven la RA como una forma de acceder a experiencias visuales sorprendentes incrementando la motivación por aprender.

En este sentido, la investigación se muestra relevante desde el punto de vista práctico, dado que se propone un recursoinstruccional apoyado en la realidad aumentada para el desarrollo de los contenidos de estructuras del área de tecnología en los estudiantes de séptimo grado de la institución Educativa Colegio "Pablo Correa León", ubicada en el municipio de Cúcuta, ello permitirá el desarrollo de acciones dentro de los espacios escolares que permitan la mejora del trabajo del docente de tecnología centrado en este caso en la enseñanza de la estructura, de igual manera impactará de manera positiva en la construcción de aprendizajes significativos en la población estudiantil.

Desde el punto de vista teórico, la investigación se muestra relevante, dado que centra su atención en función de mecanismos inherentes a la adopción de fuentes de primera mano, para de esta manera lograr un impacto significativo en la construcción conceptual del objeto de estudio, de igual forma, se espera que la información aquí presentada servirá de antecedente a otros estudios enmarcados en lo propuesto, además de ello se convertirá en un recurso bibliográfico de consulta para aquellos interesados en abordar el tema planteado.

En el mismo orden de ideas, el estudio se justifica desde el punto de vista metodológico, dado que la naturaleza del objeto de estudio, permitirá el diseño de instrumentos de recolección de la información que permitan obtener datos veraces que definan el comportamiento del objeto de estudio dentro de la realidad definida para tal fin.

\section{Materiales y métodos}

Asumir el desarrollo de una investigación en ciencias sociales, implica el abordaje de fenómenos que atañen necesariamente dentro de la misma dinámica de los contextos sociales, surge el abordaje, desde una perspectiva cualitativa y otra desde una perspectiva cuantitativa, en el caso del presente estudio, la autora considera pertinente la selección del paradigma cuantitativo, el cual es definido por Hernández, Fernández y Baptista (2006) de la siguiente manera: "Implica la medición de variables cuyas descripciones se asumen corno correspondiente al estado de las cosas y se fundamenta en la teoría de las posibilidades, esto es, la posibilidad de aplicar los 
resultados en circunstancias generales". (p. 56).

El abordaje de la investigación cuantitativa, atiende al desarrollo de elementos propios de la realidad, mediante la medición de estos, en atención a ello, se establecen variables, en el caso propio de la presente investigación, se trabajará con la variable: material instruccional, realidad aumentada y los contenidos de estructuras del área de tecnología en los estudiantes de séptimo grado de la institución Educativa Colegio "Pablo Correa León”, ubicada en el municipio de Cúcuta.

Con la finalidad de desarrollar este paradigma dentro del presente estudio, se hace necesario asumir un tipo de investigación que permita el abordaje adecuado del objeto de estudio, como se tiene previsto el hecho de Proponer un material instruccional apoyado en la realidad aumentada para el desarrollo de los contenidos de estructuras del área de tecnología en los estudiantes de séptimo grado de la institución Educativa Colegio "Pablo Correa León", ubicada en el municipio de Cúcuta, la investigadora considera necesario el desarrollo de un proyecto factible, el mismo es tratado por el Manual de Trabajos de Grado de Especialización y Maestría y Tesis Doctorales de la Universidad Pedagógica Experimental Libertador (2010) propone:

El proyecto factible consiste en la investigación, elaboración y desarrollo de una propuesta, de un modelo operativo viable para solucionar problemas, requerimientos o necesidades de organizaciones o grupos sociales; puede referirse a la formulación de políticas, programas, tecnologías, métodos o procesos, el proyecto debe tener apoyo en una investigación de tipo documental, decampo o un diseño que incluya ambas modalidades. (p.21).

De acuerdo con lo anterior, un proyecto factible, atiende a la generación de una serie de fases que permiten la sistematización de los procesos que se desarrollan dentro de la investigación, de acuerdo con ello, un proyecto factible se orienta hacia la superación de las posibles necesidades que se evidencien dentro del objeto seleccionado para tal fin, de allí la importancia de desarrollar este tipo de investigación, para ello, es pertinente asumir como diseño, la investigación de campo, la cual permitirá la inserción de la investigadora dentro de la realidad, para la recolección de la información de una manera sistemática.

En este sentido, la investigación de campo es definida por Arias (2006) como aquel que "consiste en la recolección de datos directamente de la realidad donde ocurren los hechos, sin manipular o controlar variable alguna". (p. 48), al respecto la investigación de campo, logra que se muestre realmente lo que está sucediendo dentro de la realidad, por ello, es imprescindible, para el logro del diagnóstico del empleo de las estrategias didácticas empleadas por el docente para el desarrollo de los contenidos de estructuras y la descripción de herramientas y recursos que desde la tecnología de realidad aumentada pueden aplicarse en el desarrollo de los contenidos de estructuras.

\section{Fases de la Investigación}

Fase Diagnóstica: El diagnóstico del estudio, atenderá a diagnosticar las estrategias didácticas empleadas por el docente para el desarrollo de los contenidos de estructuras y describir las herramientas y recursos que desde la tecnología de realidad aumentada pueden aplicarse en el desarrollo de los contenidos de estructuras, además de lograr la inserción del aparato conceptual para de esa manera lograr un impacto adecuado dentro de la comunidad objeto de estudio.

Fase de Factibilidad: Para el desarrollo de esta fase, se partirá por determinar la factibilidad de aplicación de un material instruccional apoyado en la realidad aumentada para el desarrollo de los contenidos de estructuras del área de tecnología en los estudiantes de séptimo grado de la institución Educativa Colegio "Pablo Correa León", ubicada 
en el municipio de Cúcuta, la misma se prevé desde el plano técnico, político, institucional, educativo, pedagógico y económico.

Fase de Diseño: Para el desarrollo de esta fase, se tiene previsto el diseño de un material instruccional para el desarrollo del contenido de estructuras mediante la aplicación de la realidad aumentada en el área de tecnología en la institución objeto de estudio.

\section{Población y Muestra}

La investigación que se pretende desarrollar, se ubica en la comunidad de la institución Educativa Colegio "Pablo Correa León", ubicada en el municipio de Cúcuta, la misma, se presenta con características propias de las comunidades educativas, en atención a ello, es pertinente definir población, al respecto Tamayo (2008), plantea que "población es la totalidad del fenómeno a estudiar, en donde las unidades de población posee una característica común la cual se estudia y da origen a los datos de la investigación" (p.114).En atención a ello, la población estará integrada por el total de estudiantes de la institución, la población estudiantil está conformada por 1326 estudiantes discriminados en diferentes grados.

Por ser una población considerablemente grande, se hace necesario fijar una muestra la misma es definida por Hernández y otros (2006) como: “en esencia un subgrupo de la población” (p.207), de manera que es necesario aplicar la selección de una serie de sujetos que permitan la generación de una información adecuada al objeto de estudio, en este sentido, la autora considera preciso la selección de la muestra del tipo proporcional al azar, la misma es definida por Castañeda (2006) como aquel que consiste "en seleccionar los individuos totalmente al azar... La muestra al ser estratificada, implica una subdivisión de la misma en grupos o estratos más pequeños" (p. 83). En este sentido, dicha cantidad radica en que esta es accesible a la autora de la presente investigación, de acuerdo con ello, se seleccionará a 82 sujetos sin distingo de sexo o de oficio.

\section{Procedimiento para la Recolección de Datos}

Asumir el proceso para la recolección de la información, hace énfasis en la generación de todo un protocolo de desarrollo para tal fin, asumiendo los instrumentos y las técnicas de recolección de la información, al respecto Ballestrini, (2001) indica acerca de este aspecto: "el conjunto de técnicas que permitirán cumplir con los requisitos establecidos en el paradigma científico, vinculados al carácter específico de las diferentes etapas de este proceso investigativo y especialmente referidos al momento teórico y metodológico de la investigación" ( $p$. 131). En atención a ello, se asumirá el diseño de un cuestionario estructurado, el cual poseerá una serie de ítemes, en correspondencia con los indicadores establecidos en la operacionalización de las variables, por ello, se asumirá un cuestionario bajo la acepción escala tipo likert

Al respecto Sabino (2002), manifiesta que el diseño del cuestionario, tipo escala: "es exclusivo de las ciencias sociales y se basa principalmente, en un acopio de datos obtenidos mediante consultas o interrogatorios referentes a estados de opinión de cualquier aspecto de actitud humano" (p. 135). En este sentido, cada uno de los planteamientos poseerá una serie de categorías de respuesta, dentro de las cuales destaca: siempre, casi siempre algunas veces, casi nunca y nunca, cada una de ellas permitirá evidenciar la frecuencia con la cual suceden los indicadores que se registran en la definición de la variable.

\section{Validez y Confiabilidad}

Dentro de la presente investigación, se hará mención exclusiva a la validez del instrumento, la misma es ejecutada, de acuerdo con los postulados de Ruiz (2002); mediante la técnica "juicio de expertos" esta técnica permite que se observe y valore el instrumento desde tres ópticas diferentes, como es el caso de un experto en tecnología, un 
experto en material instruccional y un experto en metodología, a estos se les presentará el título del trabajo, los objetivos del estudio, la operacionalización de las variables, el instrumento de recolección de la información y el formato de validación, donde estos registraran la evaluación, estas consideraciones serán acatadas por la investigadora, para el diseño de la versión final del instrumento.

Para el establecimiento de la confiabilidad se procederá de la siguiente manera; (a) aplicación de una prueba piloto, (b) codificación de las alternativas de respuestas, (c) trascripción de respuestas en una matriz de tabulación de doble entrada con el apoyo del programa computarizados Excel y (d) Calculo del coeficiente del Alfa de Crombach y e) interpretación de los valores tomando en cuenta la escala sugerida por Ruiz (2002): 0.81-1 = Muy Alta, 0.61- $0.80=$ Alta, 0.41 - $0.60=$ Moderada, $0.21-0.40=$ Baja y $0.01-0.20$ = Muy baja.

\section{Procesamiento y Análisis de Datos}

Una vez concluida la recolección de los datos se procederá a realizar las siguientes actividades: (a) revisión de cada uno de los cuestionarios para verificar que fueron respondidos en su totalidad; (b) elaboración de una matriz de datos donde se plasmaran las respuestas emitidas por los docentes en cada uno de los ítems, los cuales serán previamente codificados, tomándose como el valor más alto (5) la opción favorable y el valor más bajo (1) como la opción desfavorable (c). Se realizaran cuadros descriptivos donde se indicaran las alternativas de respuesta de cada Ítem con sus respectivas frecuencias simples y porcentuales y (d) se procederá con el análisis de cada indicador y dimensión, retornando aspectos teóricos considerados en el estudio para reforzar su interpretación.

\section{Resultados y discusión}

En la actualidad la tecnología, se ha apoderado de la educación de una manera vertiginosa y la cual debe ser aprovechada para facilitar el proceso de enseñanza y aprendizaje, el área de tecnología es muy importante para preparar a los estudiantes y que puedan egresar de las instituciones educativas para enfrentarse con cualquier realidad que se les presente, en el presente todo lo que está alrededor del ser humano tiene que ver con recursos tecnológicos, el uso de redes sociales, conexión a internet, entre otros dispositivos que permiten comunicarse e informarse, es por ello que se hace necesario realizar investigaciones que permitan por medio de la tecnología dar posibles soluciones a las problemáticas que se encuentran presentes en las instituciones educativas, a continuación se presentan los resultado del presente estudio, el cual se realizara a través de las dimensiones.

\section{Dimensión Presentaciones con Ordenador}

Es necesario que durante el acto pedagógico se tenga el pleno dominio de manejar la información a través de presentaciones en los computadores, bien sea el manejo dentro del aula de tecnología o a través del uso de un proyector multimedia, que permita que a través de materiales audiovisuales los estudiantes obtengan un aprendizaje significativo, en el presente esta estrategia es muy usada, pues las instituciones educativas han sido dotadas de equipos de computación y audiovisuales que facilitan el proceso de enseñanza, y así motivar a los estudiantes el uso de la tecnología y la importancia de ella, es necesario resaltar que en la actualidad los jóvenes tienen un control total de la tecnología, pero es necesario que sean educados para darles un uso adecuado, en esta dimensión se midieron tres indicadores los cuales son; exposición, explicación y razonamiento.

Con respecto a los tres primeros ítems, después de haber realizado en análisis a través de tablas de frecuencia y realizar la representación gráfica es necesario realizar el análisis descriptivo de cada uno de los ítems de la dimensión presentación en ordenador,donde se pregunta en el Ítem 1: ¿El docente utiliza exposiciones como estrategia 


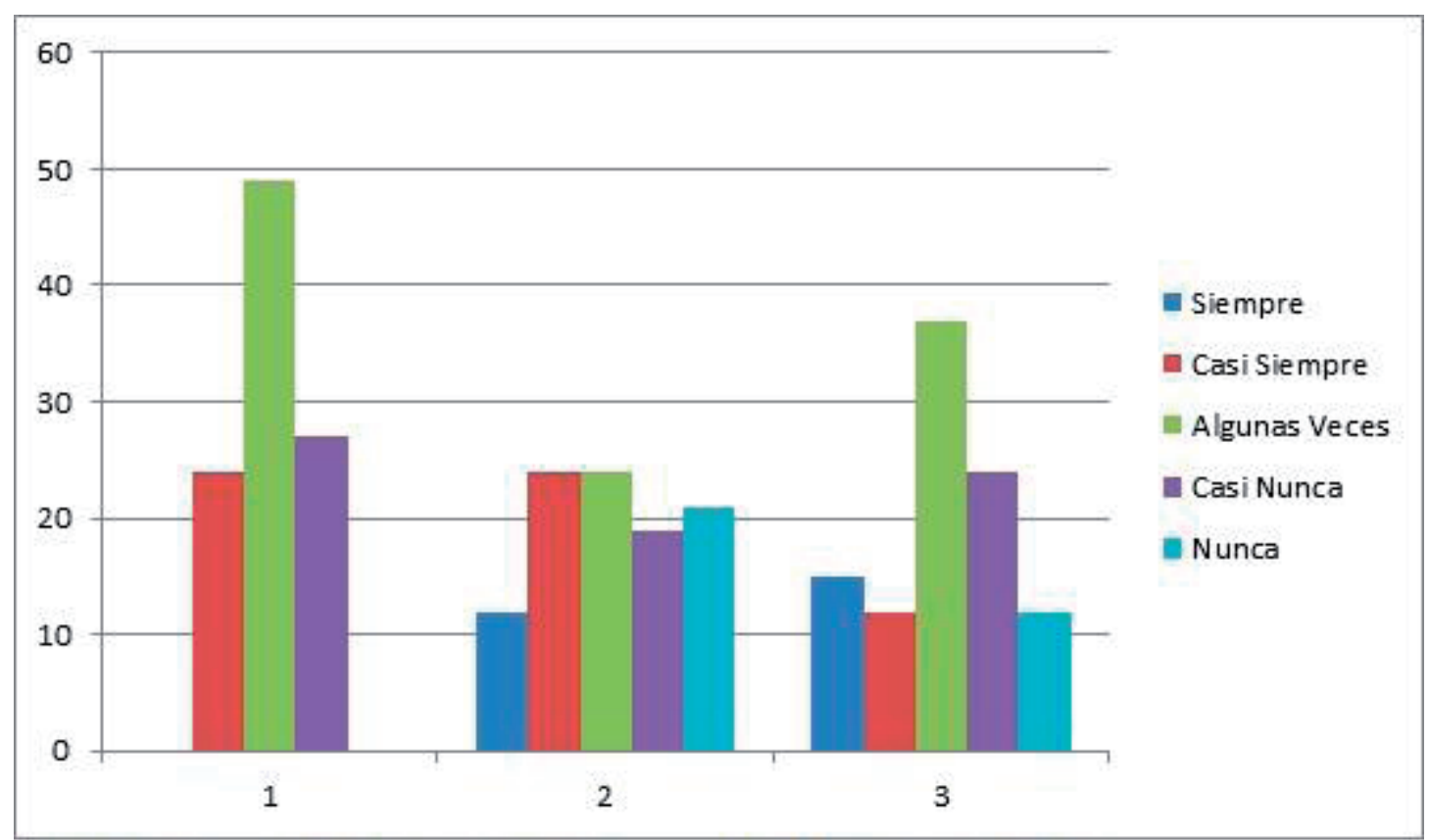

Gráfico $\mathrm{N}^{\circ}$ 1: Dimensión Presentación en Ordenadores. Fuente: Instrumento aplicado.

para la explicación del contenido?, el 24\% respondieron que casi siempre el docente utiliza como estrategia de enseñanza la exposición, con apoyo a proyecciones multimedia, esta técnica es muy importante, pero se puede notar que se tienen el concepto errado de una exposición cuando se utiliza como estrategia de enseñanza, debe ser llamativa, con el uso de colores suaves y preferiblemente enfocados en mapas mentales o conceptuales con el fin de que a simple vista se entienda lo que quiere explicar y el estudiante capte de una manera más interactiva.

Asimismo un 49\% dieron como respuesta algunas veces, pues por lo general se considera que una exposición es pararse al frente y hablar y hablar del tema sin importar si de verdad el mensaje que se desea entregar está llegando de una manera productiva a los estudiantes, así que es necesario cambiar ese concepto y realizar exposiciones primero que todo con ayuda de recursos tecnológicos que permiten captar el interés de los estudiantes y logren así obtener un aprendizaje significativo de los contenidos se están explicando, asimismo es importante destacar que cuando se trata de teoría para llevar a la práctica, una exposición efectiva es ideal para lograr el conocimiento en los estudiantes.

De la misma manera el $27 \%$ restante de los encuestados respondieron casi nunca, pues el docente se enfoca en el pizarrón y la proyección pero no tienen la facilidad para explicar a través de una exposición y más cuando se trata de áreas como tecnología que la misma tienen contenidos como programación, algoritmos, bases de datos entre otros, es necesario que el docente utilice recursos tecnológicos y a su vez sea más pedagógico en el momento de dar esta asignatura que para ser entendida es necesario que se presente la didáctica ese enlace entre lo teórico y lo práctico.

Desde otro punto de vista, se presenta el ítem $\mathrm{N}^{0} 2$ el cual expresa; ¿Cree usted que el docente explica bien cada una de sus clases?, el $12 \%$ respondieron que siempre, el docente debe saber motivar al grupo de estudiantes, captar su atención y tener un discurso con mucha seguridad para así lograr dar una buena explicación de sus clases, algunos profesores tienen gran conocimiento pero no tienen la pedagogía necesaria para lograr que el grupo de jóvenes que está formando puedan 
obtener el conocimiento necesario, es por ello que se hace necesario que el docente este actualizado $\mathrm{y}$ sobre todo que tenga un control total de las actividades a desarrollar en el aula de clase. En el mismo orden de ideas un $24 \%$ respondieron que casi siempre, el docente explica, cuando las áreas son teórico - prácticas es necesario que el docente muestre seguridad en el dominio de los contenidos así como el manejo de los recursos para poder explicar de una manera efectiva y logre que el estudiante obtenga un aprendizaje significativo, pues en el área de tecnología, que es una materia que capta el interés de los jóvenes si no se maneja la interacción, los recursos, las técnicas, las estrategias se hace un poco tedioso en acto pedagógico y más cuando se tratan de algoritmos, bases de datos, estructuras entre otros contenidos que en la actualidad se le exige al estudiante para su egreso.

Asimismo otro $24 \%$ dieron como respuesta algunas veces, el joven del presente son nativos digitales, su naturaleza es el manejo de los recursos tecnológicos, bien sean celulares, computadores, Tablet, software, redes sociales entre otros, los cuales de manera empírica tienen el dominio total de cada uno de ellos, es por eso que cuando ellos nota que el docente solo se enfoca en dictar o copiar en la pizarra, ya pierden el interés, aquí entra el juego del docente que debe prepararse para que cada encuentro pedagógico el joven se sienta motiva y logre mantenerse motivado, interesado en querer aprender más y más con relación a los contenidos.

Ahora bien, otro 19\% respondieron que casi nunca, la explicación de docente cubre las expectativas de los estudiantes y más cuando se trata de lo teórico, pues la práctica es diferente, también dependiendo del contenido que se está desarrollando si son ejercicios de programación es necesario que exista un poco de didáctica, dejar a un lado el régimen direccional y hacer de una manera más flexible cada una de las explicaciones utilizando recursos tecnológicos y lograr que los jóvenes reflexionen y comprendan cada uno de los ejercicios de los contenidos expuestos.

El $21 \%$ restante de los encuestados respondieron que nunca, pues ellos consideran que el docente del área de tecnología tiene el dominio de lo teórico pero cuando tiene que desarrollar los ejercicios o la explicación con números binarios se queda corto en la explicación y no logra que los estudiantes entiendan los ejercicios que él explica en el pizarrón, pues es de acotar que aunque ven las clases en el salón de informática ellos aun utilizan el pizarrón allí para dar la explicación. es recomendable que los docentes se mantengan actualizados con respecto a la didáctica y la pedagogía para poder desarrollar sus clases de una manera más atractiva y dinámica.

Por otra parte el ítem $\mathrm{N}^{\mathrm{o}} 3$, manifiesta; ¿Permite las explicaciones del docente que usted razone con respecto al conocimiento que se está impartiendo? el 15\% respondieron que siempre, es importante que la explicación del docente le permita al estudiante reflexionar pero sobre todo que razone y surjan cada vez más interrogantes e interés por los termas que se están desarrollando, si el docente no logra el razonamiento por parte de los estudiantes no permitirá que se forme un aprendizaje significativo, de la misma manera otro $12 \%$ respondieron que casi siempre logran razonar con lo que el docente explica, pues cuando se trata de práctica se concentran buscando el porqué de cada cosa.

En el mismo sentido se pudo observar que un $37 \%$ de los encuestados dieron como respuesta algunas veces, pues presentan dudas con respecto a las explicaciones que da el docente, no se presentan diferentes estrategias que ayuden a que los estudiantes obtengan el entendimiento de los contenidos que se están dando, es necesario que exista un dinamismo en el acto pedagógico para que el estudiante pueda razonar y obtener el aprendizaje significativo, es importante el uso de la tecnología, pues es un tema que en la actualidad está dando de qué hablar y se debe aprovechar 
durante el acto pedagógico y así lograr que los jóvenes reflexionen, análisis y razones con respecto a los temas que el docente está impartiendo.

En esta misma línea el 24\% respondieron que casi nunca, pues el docente aún no ha salido de la enseñanza tradicionalista, libros, pizarras, marcadores y no ha explorado más allá, en Colombia la mayoría de las instituciones educativas, han contado con dotaciones importantes en cuanto a tecnología, sala audiovisuales, Tablet, salas de informática que permiten un avance tecnológico en a educación ahora es responsabilidad de los directivos y docentes capacitarse y enfocarse en explorar el mundo digital para poder orientar a sus estudiantes y darles cualquier contenido con recursos tecnológicos que permitan que el estudiante razone y conozca la importancia de los mismo, y el ultimo $12 \%$ respondieron que nunca, pues en muchos casos los jóvenes se distraen con facilidad y si el docente no muestra su pedagogía manteniendo la atención del estudiante el nunca podrá obtener un aprendizaje significativo, pues no está analizando ni razonando la información que están impartiendo. (Cairo y Guardati, 2006, p. 86. Aneca, 2004, p. 32)

\section{Dimensión Ejemplos}

Esta dimensión es importante mediarla, pues el conocimiento se construye a partir de ejemplos reales que le permitan al estudiante comparar y conseguir la importancia de los conceptos, palabras, cosas, objetos entre otros, la tecnología es muy práctica y la misma se puede trabajar con entornos virtuales que lo llevan a verificar a través de ejemplos la información que se está dado, las realidades aumentadas es el mejor ejemplo a la hora de trabajar con la tecnología ya que la misma se presenta sobre espacios físicos existentes, en esta dimensión se manejaron tres indicadores los cuales son; gráficos, comprensión y aprendizaje, a continuación se presentan a través de la tabla de frecuencia.

Ahora bien, después de presentados los resultados en la tabla de frecuencia y gráficamente se procederá a realizarse el análisis de descriptivo de cada uno de los resultados del ítem $\mathrm{N}^{\circ} 4$, el cual expone; ¿El docente del área de tecnología utiliza como ejemplos gráficos?, el $73 \%$ dieron como respuesta que algunas veces, los docentes utilizan gráficos para explicar algún contenido de manera

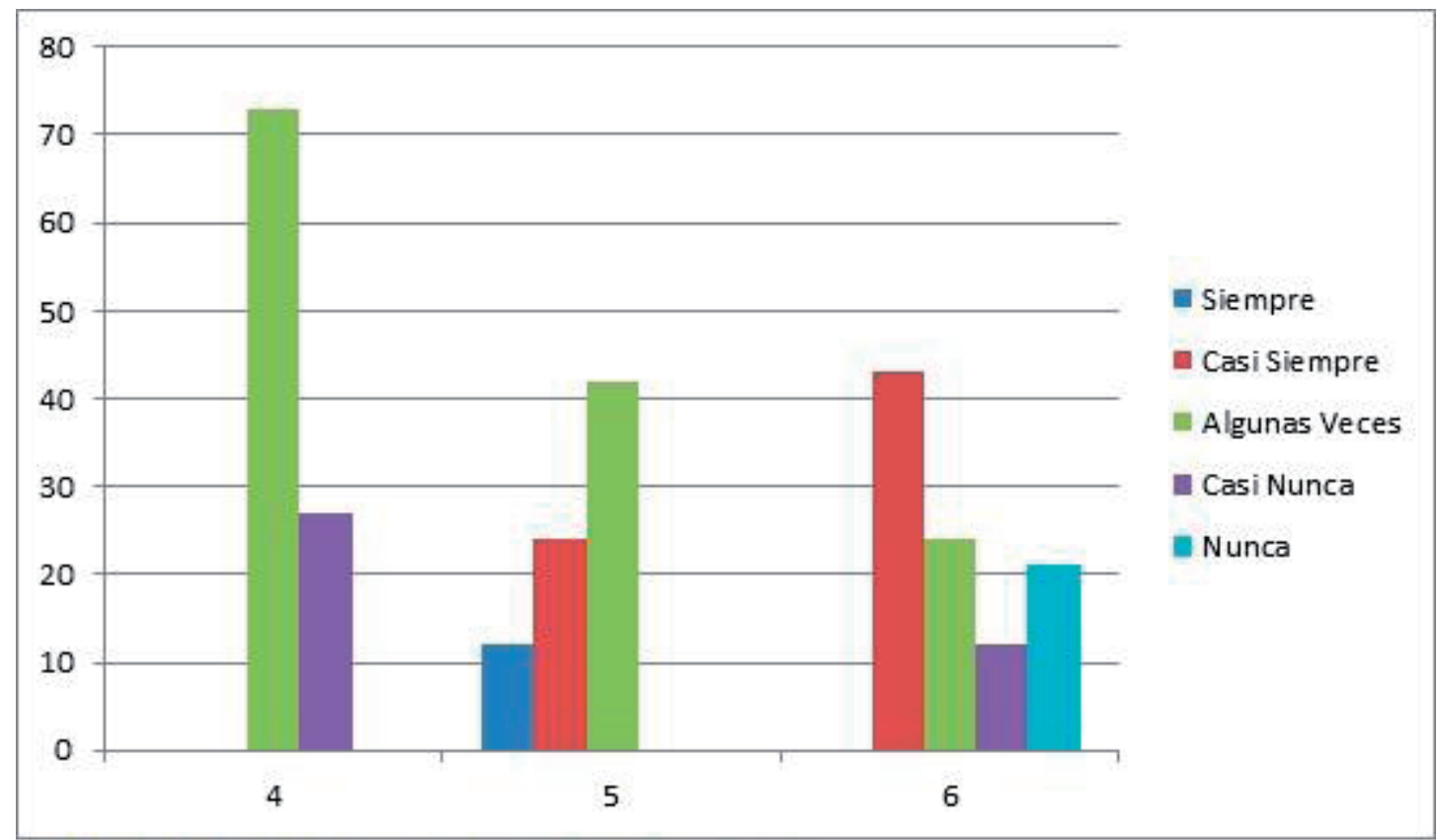

Gráfico $N^{\circ}$ 2: Dimensión Ejemplos. Fuente: Instrumento aplicado. 
práctica, es necesario la utilización de gráficos con colores llamativos y más cuando se trata de la asignatura de tecnología, pues la computadora contiene muchas herramientas que permite desarrollar gráficos y lograr que los jóvenes desarrollen la creatividad, el hemisferio izquierdo, el otro $27 \%$ restante dieron como respuesta casi nunca, pues si no se presenta un dinamismo y un manejo de herramientas adecuados, se hace imposible el uso de la gráficos como ejemplos en las estrategias que se utilizan en el acto pedagógico.

En el ítem No 5, expresa; ¿Permite los ejemplos que utiliza el docente que usted tenga la compresión necesaria del contenido?, el 12\% dieron como respuesta siempre, el docente se esfuerza para que el estudiante logre la comprensión necesaria en el momento del proceso de enseñanza, todo proceso tiene su grado de dificultad en el educativo el de formar, pues el docente debe investigar, porque el acto pedagógico no se trata solo de dictar y hablar es necesario buscar estrategias que logre que el estudiante interactúe, participe, se fomente el trabajo colaborativo y así logran la comprensión tecnológica de los estudiantes.

Otro 24\% dieron como respuesta casi siempre pues el área de tecnología, capta la atención con facilidad de los jóvenes, y algunos tienen desarrollado la inteligencia visual, la cual solo con ejemplos a través de lo visual logra internalizar el aprendizaje, pero para ello es necesario que el docente muestre y ejemplos reales y así capte la comprensión de cada uno de los estudiantes, pues en el presente la gran mayoría tienen desarrollados la capacidad del manejo de recursos tecnológicos, y comprenden a la perfección la manipulación de ellos, y el otro $12 \%$ marcaron la opción, algunas veces, es de resaltar que los docente de la institución objeto de estudio, tienen el compromiso de querer estar en constante actualización y lograr dar una educación de calidad, y el $51 \%$, que es la mayoría se inclinaron por la opción de respuesta casi nunca.

En el ítem $N^{\circ} 6$, se acota, ¿Considera usted que el docente utiliza suficientes ejemplos para que usted obtenga un aprendizaje significativo? En esta oportunidad respondieron un $43 \%$ casi siempre, pues el acto pedagógico se da a través de pequeños ejemplos que logra que el docente entrelace esto con el proceso de enseñanza y se logre un aprendizaje significativo, por otra parte otro $24 \%$ dieron como respuesta algunas veces, los ejemplos permiten que el estudiante utilice la imaginación y desarrolle capacidades que le ayudaran a comprender mejor contenidos con ciertas dificultades.

En el mismo sentido, otro $12 \%$ respondieron casi nunca, no es solo dar la teoría a través de estrategias que no contengan el enlace con los ejemplos, pues no cumplen con un proceso didáctico eficaz, es necesario el uso de los mismos, pues es importante trae acotación que el aprendizaje significativo se da cuando existe el enlace de un conocimiento previo y luego es reforzado con un conocimiento más formal, que el docente tenga la capacidad de comprender y unir estos conocimientos para que se dé el aprendizaje significativo y que mejor manera de hacerlo utilizando ejemplos claros y reales, el $21 \%$ restantes, tienen como opción de respuesta nunca, y eso está causando que los jóvenes pierdan el interés por el área de tecnología y comiencen a tenerle fobia como son las áreas de matemática, física, y otras que son tan lineales y sin procesos didácticos los jóvenes las rechazan. (Cairo y Guardati, 2006, p. 92)

\section{Dimensión Administración.}

En la actualidad, es necesario tener una administración de contenidos acorde a las exigencias de los estudiantes, pues se puede observar como los jóvenes, llamados ahora nativos digitales, tienen el control de la tecnología pero de manera empírica, y es necesario educar de una manera formal con respecto al uso de los recursos tecnológicos y sus estructura, pues no es solo lo que se puede manipular sino que en el fondo existe una programación para que los recursos 


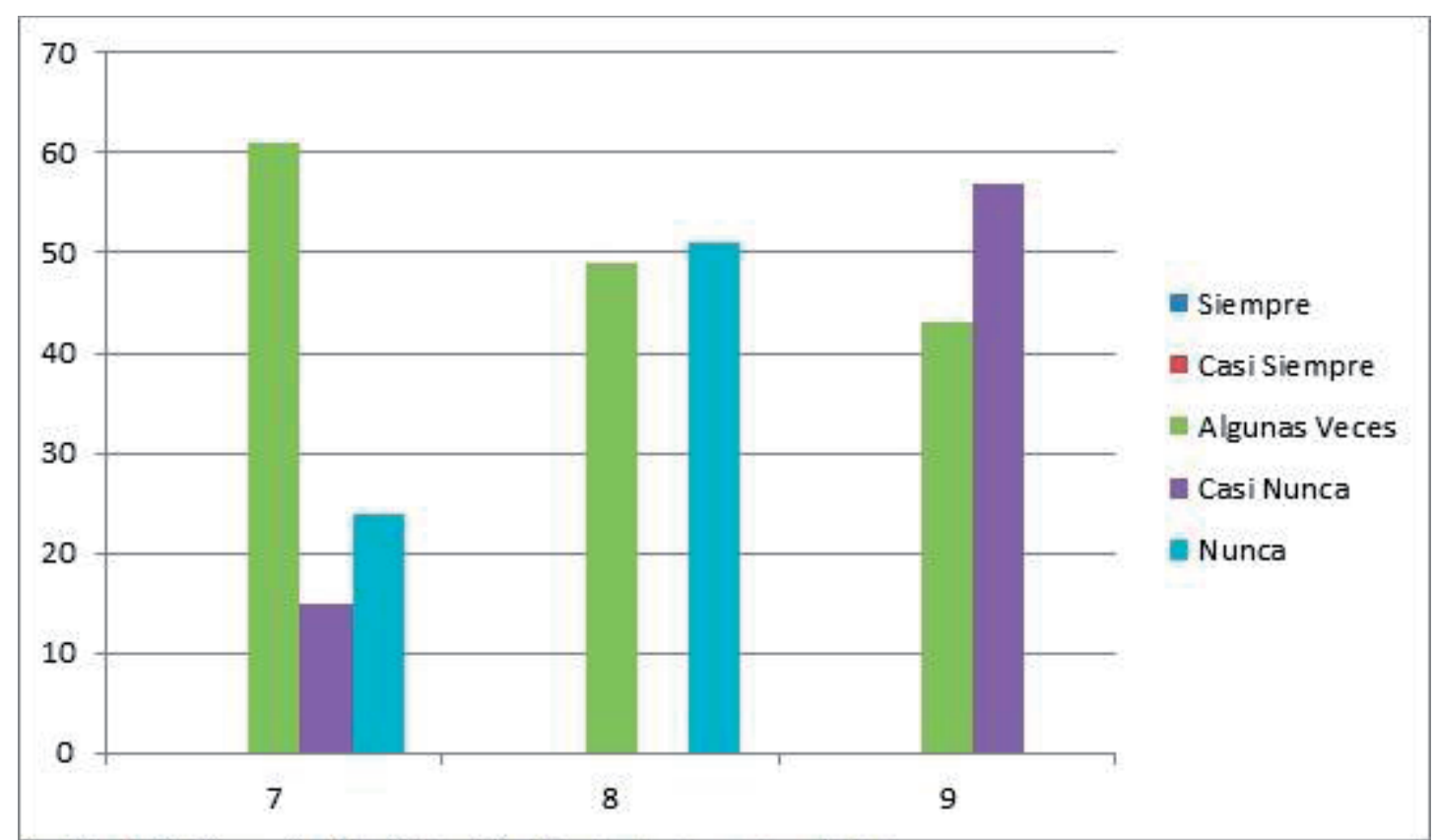

Gráfico $N^{\circ} 3$ Dimensión Administración. Fuente: Instrumento aplicado.

tecnológico, realicen las funciones para los cuales fueron programados, y el currículo juega un papel importante en la admiración de los contenidos por grados, esta dimensión contra de tres indicadores los cuales son; algoritmos, estructura de datos y conceptos, los cuales se presentaran los resultados a continuación.

El análisis descriptivo de los tres indicadores de la dimensión administración en el ítem $\mathrm{N}^{\mathrm{o}} 7$, donde se expone ¿Sabe el docente administrar estrategias para la enseñanza de los algoritmos?, un $61 \%$ respondieron que algunas veces, pues esta área de tecnología es de interés por los estudiantes, pero de manera superficial cuando comienzan a encontrar un cierto grado de dificultad el ambiente cambia y es necesario que el docente administre bien las estrategias que va a usar para que el estudiante no pierda el interés si no al contrario siga explorando e investigando cada vez más como puede realizar las cosas y así lograr alcanzar el contenido de manera satisfactoria.Asimismo otro $15 \%$ dieron como respuesta casi nunca lo que deja ver que hay poco esfuerzo por parte de los docentes de tecnología para que el estudiante pueda entender estos contenidos y logren obtener un aprendizaje significativo de los mismos, y el $24 \%$ restante dieron como respuesta nunca, es importante resaltar que el docente debe ir a diario en busca de la capacitación y así lograr investigar y estudiar las diferentes estrategias que en la actualidad se están mostrando con el fin de poder dar una educación de calidad, si es el área de tecnología, allí existe una serie de recursos tecnológicos que permiten ser utilizados con facilidad y a su vez contribuyen a la formación de un aprendizaje significativo.

En el ítem $\mathrm{N}^{\circ}$ 8, se mide, ¿El docente utiliza diferentes estrategias para administrar los contenidos sobre estructuras de datos?, en esta interrogante un 49\% dieron como opción de respuesta algunas veces, esta asignatura se desarrolla a través programación con resultados exactos para que todo funcione de una manera perfecta, así que requiere de mucho estudio, pero sobre todo de agilidad, es atractiva ante los ojos de los jóvenes, por lo tanto es necesario buscar estrategias que ayuden tanto al docente con su proceso de enseñanza como al estudiante en el de aprendizaje, estos contenidos presentan cierta 
dificultad, pero para los nativos digitales no se les hace tan difícil siempre y cuando tengan una presentación que cubra las expectativas del joven, el otro $51 \%$ dieron como respuesta que nunca, pues el docente de tecnología se vuelve un poco rígido cuando se están trabajando estos contenidos y no les dan paso a la flexibilidad, por lo que hacen que los jóvenes pierdan el interés pues el simple hecho de desarrollar los ejercicios en el cuaderno sin ir directo al computador y manipular los sistemas no se consigue que el docente logre satisfactoriamente estos contenidos.

Por otra parte el ítem $\mathrm{N}^{\circ}$ 9, manifiesta; ¿Se utiliza durante el acto pedagógico estrategias a la hora de enseñar los conceptos básicos del área de tecnología?, el 43\% respondieron que algunas veces, existe infinidad de estrategias didácticas, pedagógicas, metodológicas que permiten que se dé una enseñanza de calidad, pero si el docente no investiga es difícil que desarrolle estrategias que puedan orientar y ayudar al docente a la hora de obtener aprendizaje, es necesario que se utilicen estrategias que se debe atrás el ciclo pasivo dentro del acto pedagógico, es necesario que haya dinamismo, que el estudiante participe pero sobre todo que pueda lograr un aprendizaje significativo.

El otro 57\% respondieron que casi nunca se utilizan estrategias, también es de resaltar que en muchas ocasiones el docente no tienen seguridad de lo que está haciendo y piensa que al desarrollar una estrategia puede perder el control del grupo, lo que es algo errado, pues al contrario si el docente utiliza diferentes estrategias, va a permitir que el estudiante interactúe y se convierta en un acto pedagógico de calidad y así él se pueda sentir satisfecho con el trabajo que está realizando, las estrategias se manejan siempre de la mano con los recursos, pues no se pueden desarrollar si no cuenta con una serie de técnicas y recursos que permiten que la estrategia se muestre de una manera adecuada, en el área de tecnología se cuentan con diferentes recursos disponibles en las instituciones educativas.

\section{Dimensión Definición}

Las definiciones son de gran importancia dentro de una formación formal, pues es necesario tener un conocimiento teórico que te ayude a reforzar la práctica, de nada sirve manipular muy bien los recursos si no tienen a la mano la teórica, el significado de lo que está manejando, y aquí entra en juego la didáctica la cual es ese enlace ente la teoría y práctica, para que exista un aprendizaje significativo, esta dimensión está conformada por dos indicadores los cuales son: visión y dispositivos tecnológicos, con base importante en el manejo de estrategias para lograr integrar las definiciones o conceptos de una manera interactiva en el acto pedagógico, a continuación se presentan los resultados de esta dimensión a través de la tabla de frecuencia, la representación gráfica y el análisis descriptivo correspondiente por cada ítem.

Con respecto a lo anterior, ahora se presenta un análisis descriptivo de estos dos ítems comenzado por el $\mathrm{N}^{\circ} 10$ el cual expresa; ¿El docente utiliza con frecuencia recursos visuales en la explicación de contenidos?, en el mismo dieron como respuesta un $37 \%$ la opción casi siempre lo cual deja ver que no frecuentemente el docente se esmera por realizar una clase de calidad, y menos que los jóvenes entiendan el contenido que está explicando, se ha comprobado que todo lo que se muestra de una manera creativa realiza un cambio en el ambiente donde se desarrolle, es por ello que se hace necesario en un aula de clase trabajar recursos visuales que permitan estimular la creatividad del joven y a su vez comprenda el significado de lo que se le está enseñando.

Por otra parte otro $37 \%$ respondieron que algunas veces, es importante el uso de recursos visuales que permitan captar la atención del joven por más tiempo, en la actualidad mucho es el material que se puede utilizar como recurso visual, como; videos, proyecciones multimedia, foros, entre otros que ayudan a fortalecer el proceso de enseñanza y el otro $26 \%$ respondieron que nunca, aun se puede notar como los docentes tienen miedo 


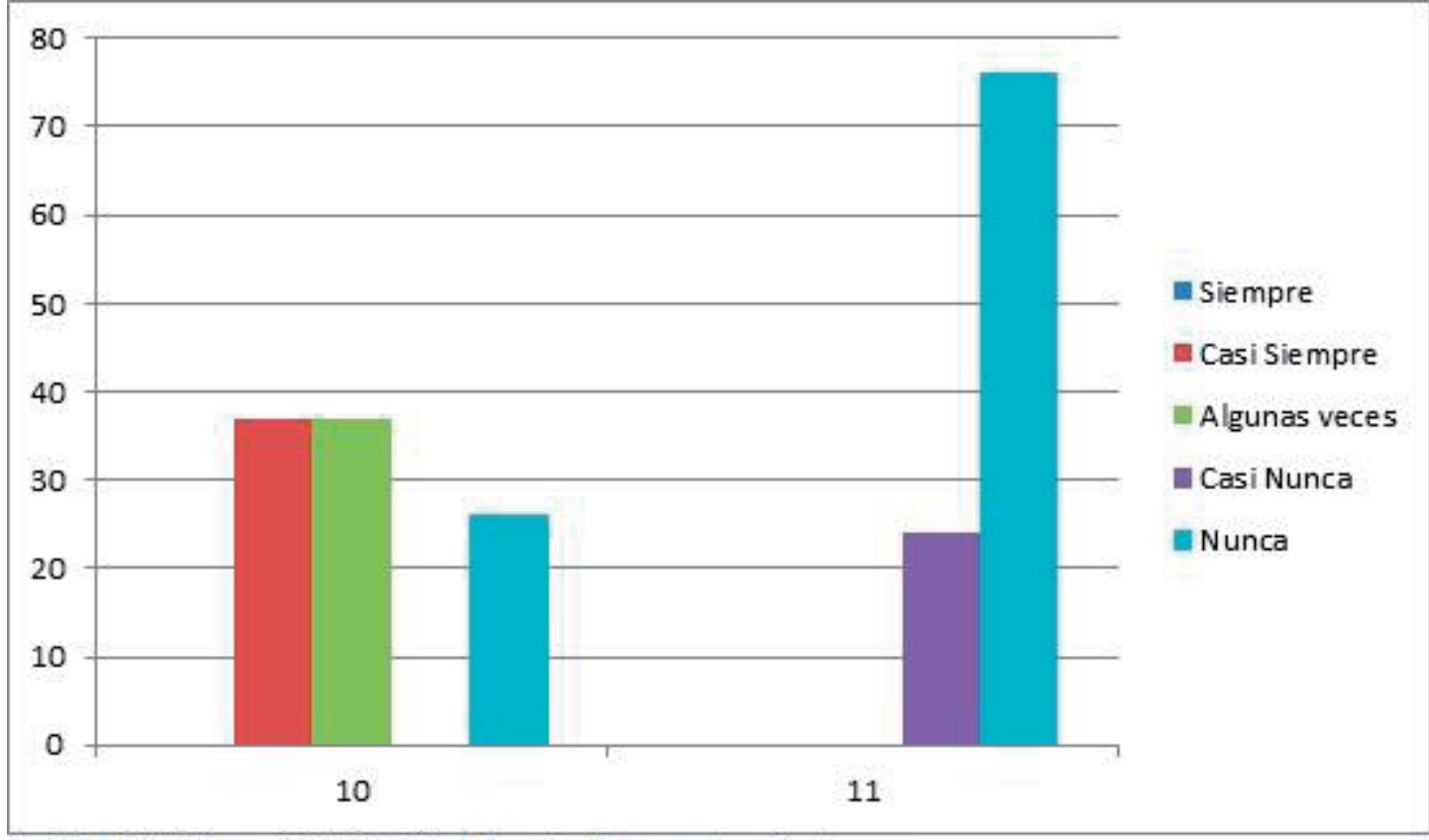

Gráfico $\mathrm{N}^{\circ} 4$. Dimensión Definición. Fuente: Instrumento aplicado.

al cambio, prefieren no usar nuevos recursos, que prepararse y enfrentar la realidad, para poderle brindar a los jóvenes una educación de calidad, y en la actualidad son muchos los espacios de información que muestran cómo usarlos, la manipulación y la importancia de cada uno de ellos.

El ítem $\mathrm{N}^{\mathrm{o}} 11$, expone; ¿Considera usted que se le da el uso necesario a los dispositivos tecnológicos como recurso de enseñanza?, en esta oportunidad el $24 \%$ respondieron que casi nunca, es importante resaltar que hasta con el celular que los jóvenes tienen se pueden utilizar como recurso de enseñanza y así los jóvenes entiendan que el celular no solo es para enviar y recibir mensajes, llamadas y jugar si no que se le puede dar otro uso educativo y se pueden formar de una manera más divertida y responsable, el otro $76 \%$ respondieron que nunca, se usan los dispositivos tecnológicos en alguna clase o actividad educativa, por lo que deja ver que los docentes se enfocan en otros recursos dejando a tras los más importantes y con los que cuentan al alcance de todos los docentes y estudiantes.

\section{Dimensión Usos Educativos}

En la educación, han surgido muchas estrategias, técnicas, recursos que permiten que el proceso de enseñanza resulte con más facilidad que anteriormente, es por ello que se encuentran software educativos a los cuales se les da un uso especial, asimismo a simuladores, traductores, todo a la mano de las personas que están interesadas por aprender más sobre la tecnología, desde el punto de vista educativo, son muchos los usos que se le dan a los recursos tecnológicos y quizás creados con fines empresariales pero en la actualidad se han ido adaptando de una manera vertiginosa en la educación y hasta el presente se ha podido solucionar muchas de las problemáticas existentes en la educación por medio de la tecnología y el uso de recursos tecnológicos, en esta dimensión se encuentran cuatro indicadores los cuales son; Libros con modelos 3D, simulaciones, traductores en tiempo real, asistencia a la realización de tareas, accesibilidad, a continuación se presentan los resultados.

A continuación se presenta en análisis 


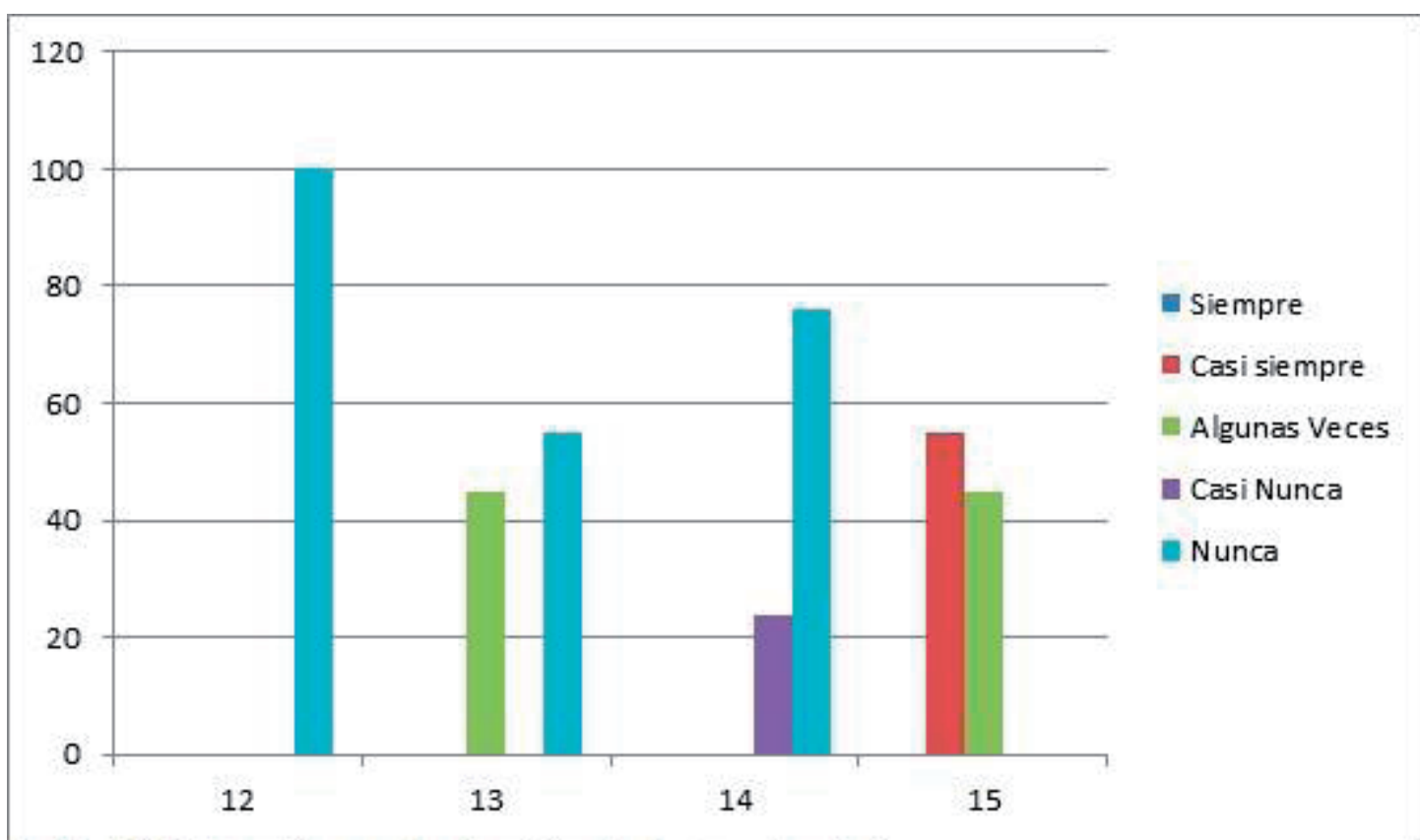

Gráfico $\mathbf{N}^{\circ}$ 5. Dimensión usos educativos. Fuente: Instrumento aplicado.

descriptivodelosítemsanteriormenterepresentados en la tabla de frecuencia y gráficos, el $\mathrm{N}^{\mathrm{o}} 12$, manifiesta ¿Considera usted que se manejan libros en modelos 3D para el aprendizaje significativo?, el esta oportunidad el $100 \%$ se inclinaron por la opción de respuesta nunca, pues los docentes no se enfocado en usar educativamente este medio como es el uso de los libros en 3D, los cuales con innovadores y permiten que el joven explore y por sus propios medios se sienta motivado a usar este recurso como manera de formarse, el internet no se trata solo de revisar información sin autor es necesario la revisión de temas bibliográficos y más cuando se trata de tecnología innovadora como son los libros en 3D.

Asimismo, se presenta el ítem Nº13, ¿Maneja programas como simuladores en la practicas de contenidos?, el $45 \%$ respondieron que algunas veces, es de resaltar que las instituciones educativas cuentan con simuladores en algunas áreas en especifica que en muy pocas oportunidades son utilizadas por los docentes, es necesario la capacitación por parte de los docentes con el fin del uso de estos equipos y así puedan incentivar a los jóvenes a usarlos de una manera adecuada, el otro 55\% respondieron que nunca, pues aunque en el estado han dotado de estos equipos a las instituciones no se usan y se guardan bajo cierto recelo por parte de los directivos.

Seguidamente se presenta el ítem $N^{\circ} 14$ el cual expone; ¿Se utiliza a menudo traductores en tiempo real para aclarar dudas?, el 24\% respondieron que casi nunca, pues los traductores en la actualidad son necesarios ya que mucha información con respecto a la tecnología se presenta en ingles por lo cual es necesario que cuando el estudiante tenga dudas con respecto a un significado es necesario utilizar un traductor en tiempo real que cubra las expectativas del estudiante y porque no del docente, los equipos de computación que se encuentren en el área de tecnología deberán tener un traductor adecuado que permita aclarar dudas, el otro $76 \%$ respondieron que nunca, pues es muy poca la importancia que se le da a los términos que se encuentran en otro idioma y que puede ser de gran importancia para la formación.

El ítem No 15, expresa; ¿Asiste continuamente a la realización de tareas referentes a la tecnología?, el 55\% respondieron que casi siempre, pues se sienten motivados por parte de los docentes, 
es necesario que el joven este en constante manipulación de los recursos tecnológicos, para que de esta manera se pueda preparar de una manera más eficiente, si solo lo hace en las horas de clase no vera resultados positivos en la formación, el otro $45 \%$ respondieron que algunas veces, pues por el miso dinamismo en que se presenta $\mathrm{v}$ el acto pedagógico, los estudiantes van perdiendo el interés, y toma muy poca importancia en cumplir con cualquier actividad que le sea asignada.

\section{Dimensión Impacto de la Realidad Aumentada}

En la actualidad han surgido temas dentro de la tecnología, que han llamado la atención de muchas personas, este es el caso de la realidad aumentada la cual ha tenido un gran impacto en la sociedad, en diferentes áreas y es hora que entre a la educación de una manera más formal y que mejor manera de hacerla que ayudando a facilitar el proceso de enseñanza en el área de tecnología, pues permite a través de recursos tecnológicos y el espacio físico abrir un mundo virtual que es muy seguro que los jóvenes tomen curiosidad por introducirse en este medio y explorar cada vez, es necesario aprovecharlo y orientar a los jóvenes con respecto a este tema la dimensión consta de tres indicadores los cuales son; aprendizaje significativo, motivación e integración, a continuación se mostraran los resultados.

Con respecto a lo anterior se presenta el análisis descriptivo de estos ítems el $\mathrm{N}^{\mathrm{o}} 16$, manifiesta; $¿$ Cree usted que hay accesibilidad a la realidad virtual en la institución?, en esta oportunidad del $100 \%$ respondieron que algunas veces pues en muchas instituciones educativas el gobierno lo ha dotado con internet pero los directivos para no perder el control de los estudiantes, prefieren tenerlos restringido, y los docentes por no llevarle la contraria de los directivos prefieren seguir planificando con lo que estén a su alcance sin llevar a los estudiantes a conocer la realidad virtual con fin educativo y más en el área de tecnología donde se presentan contenidos con un grado de dificultad alta.

En este mismo sentido se encuentra el ítem $\mathrm{N}^{\mathrm{o}}$ 17, el cual expone; ¿Permite la realidad aumentada obtener un aprendizaje significativo?, el 55\% respondieron que casi siempre, pues la gran mayoría han tenido acceso a la realidad aumentada

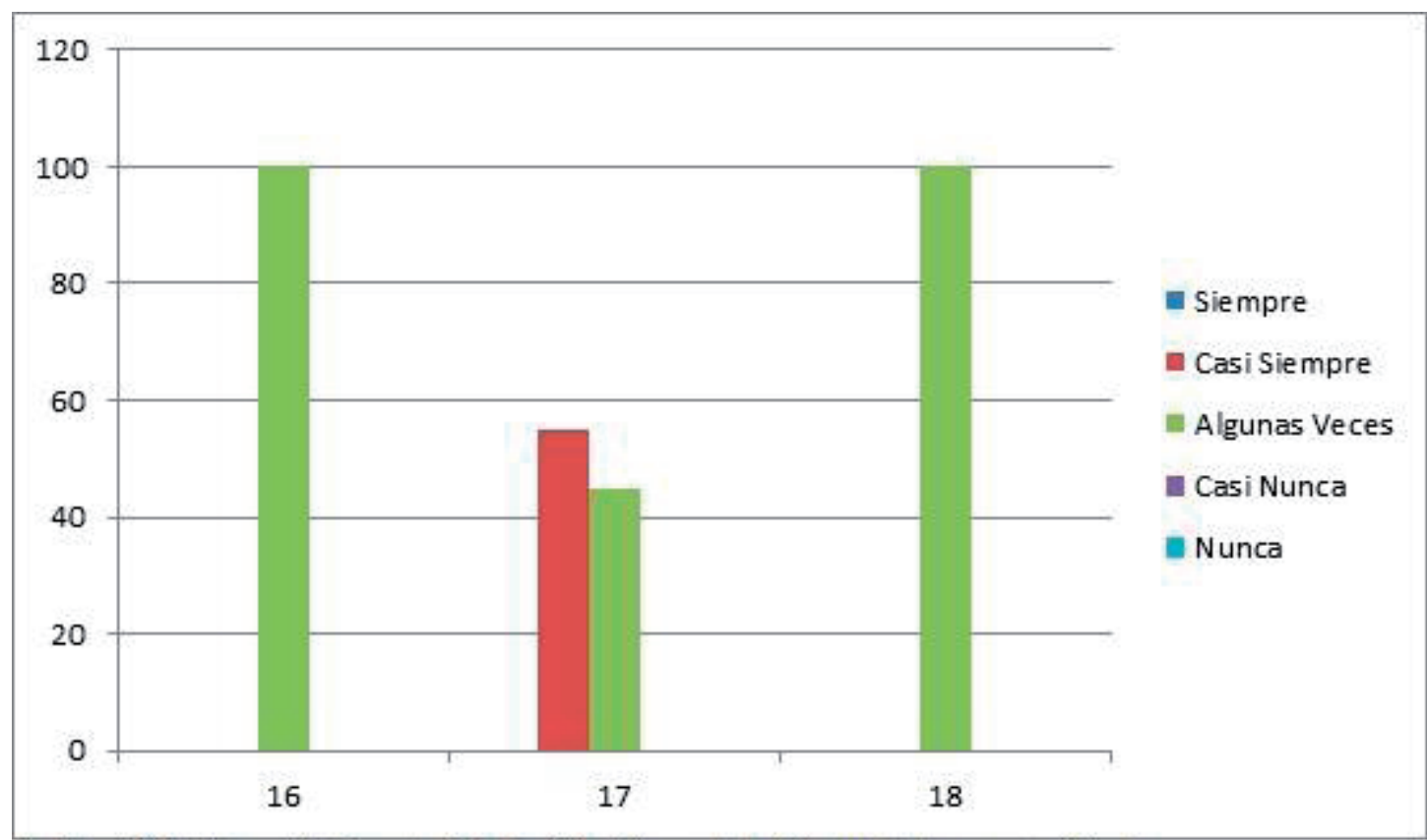

Gráfico $\mathrm{N}^{\circ}$ 6. Dimensión Impacto de la Realidad Aumentada. Fuente: Instrumento aplicado. 
por medio de juegos que han promovido esta programación, la realidad aumentada es el trabajo de espacios que al ser utilizados en la educación, esto promovería un conocimiento grande y haría que los jóvenes se sientan motivados, pero sobre todo que mantenga el interés en estos temas nuevos que han evolucionado el mundo, el otro $45 \%$ respondieron que algunos veces, pues no conocen el manejo exacto de esta realidad pero se les puede observar el interés por este tema.

El ítem No 18, indica, ¿Cuándo el docente utiliza como estrategia el trabajo con la realidad aumentada usted se siente motivado?, en esta oportunidad el $100 \%$ se inclinó por la opción de respuesta algunas veces, pues en realidad se pudo observar que en muy pocas oportunidades han tocado el tema de la realidad aumentada y los jóvenes solo lo conocen de una manera muy superficial es importante orientar a los jóvenes y capacitarlos a través de la realidad aumentada, para que se motiven a trabajar cualquiera de los contenidos o en este caso por ejemplo lo de estructuras de datos que se presentan de una manera no muy fácil para el trabajo con ellos, es por esto que al trabajar espacios físicos como la programación a través de un espacio aumentado con dispositivos tecnológicos le abrirá las puertas para una educación de calidad.(Ruiz, 2011, p. 31, Bimber y Raskar, 2005, p. 230, Espinoza, 2015, p. 13, Espinoza, 2015, p. 16)

\section{Dimensión Factibilidad}

Es importante conocer la factibilidad de la propuesta que se quiere diseñar y así poder obtener mejores resultados a la hora del diseño la realidad aumentada es un tema nuevo pero muy bien sustentado y utilizado en el presente se ha visto como personas han creado diferentes cosas con la realidad aumenta por ejemplo los grandes eventos deportivos se ha visto como esta tecnología se apoderado y ganado espacio, los juegos los cuales han causado grande controversia por el alcance que han presentado es por esto que se hace necesario conocer la factibilidad del uso de la realidad aumentada en el proceso educativo y que resultados puede prometer, la misma cuenta de tres indicadores los cuales son; técnica, educativa y pedagógica, a continuación los resultados.

Después de presentar el análisis estadístico se presentara el descriptivo, con el fin de cumplir metodológicamente con la presentación de los resultados, el ítem $\mathrm{N}^{\mathbf{1}} 19$ indica; ¿Considera usted que es factible utilizar como técnica la realidad aumentada en el proceso de enseñanza?, el $76 \%$ respondieron que siempre, pues es importante reconocer que la tecnología avanza a paso agigantado y no se puede quedar solo en la transcripción de un texto o la proyección de un video es necesario buscar otras estrategias innovadoras que permitan tanto al docente como al estudiante, estudiar e ir más allá de lo que se ve a simple vista, el otro $24 \%$ marcaron como opción de respuesta casi siempre, pues están ansiosos por conocer cada día con respecto al tema de la realidad aumentada.

En el ítem $\mathrm{N}^{\mathrm{o}}$ 20, expone, ¿Mejoraría el proceso educativo al facilitar la enseñanza y el aprendizaje del área de tecnología?, el 100\% respondieron que siempre pues el proceso educativo está abierto a constantes cambios claro esta para mejorar y al conseguir que el proceso de enseñanza y aprendizaje se desarrollen de una manera más eficiente automáticamente mejora la calidad de la educación y se le estaría brindado al estudiante el desarrollo de capacidades y habilidades tecnológicas que pueden ser utilizadas en un futuro cuando deseen desenvolverse en un ámbito laboral.

Y para finalizar se encuentra el ítem $\mathrm{N}^{\circ} 21$ el cual acota; ¿Durante el acto pedagógico el docente utiliza estrategias didácticas para facilitar el proceso de enseñanza?, en esta oportunidad un $55 \%$ respondieron que algunas veces, es importante resaltar que en la tecnología existen muchos recursos que permiten facilitar el proceso de enseñanza, pero se deben utilizar de una manera 


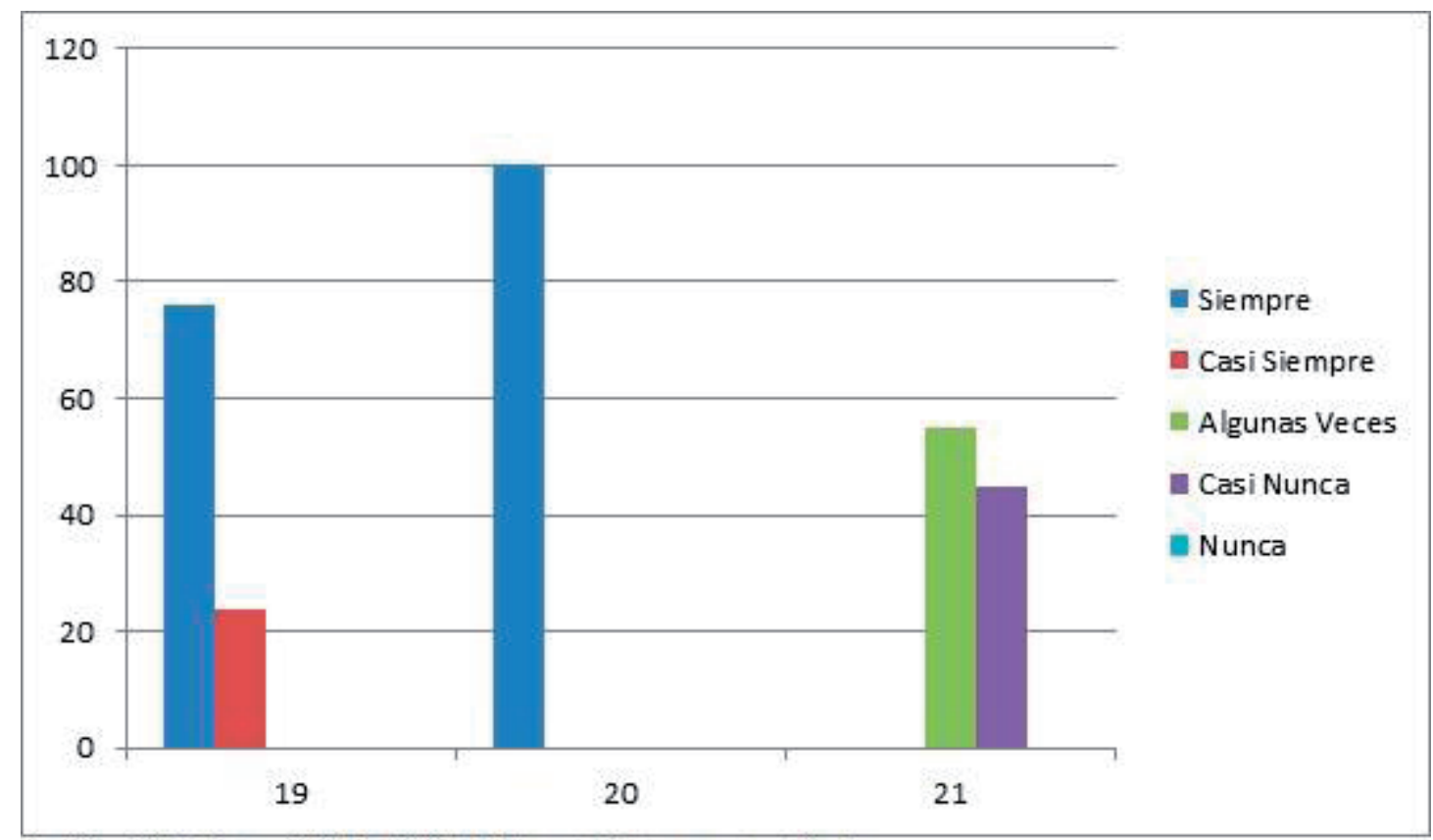

Gráfico $N^{\circ}$ 7. Dimensión Factibilidad. F uente: Instrumento aplicado.

ordenada, y con todos los pasos que se deben seguir como es organizar, seleccionar, planificar y tener el control de las mismas, el otro $45 \%$ respondieron que casi nunca, no solo se debe desarrollar el acto pedagógico, el joven frente al computador desarrollando talleres sencillos, si no que se deben implementar estrategias que sirvan para que los jóvenes capten la información de una manera más eficiente.

\section{Conclusión}

La tecnología en la educación ha sido participe de muchos cambios positivos, pues ha generado en los niños, jóvenes y adultos un sinfín de conductas que han permitido el crecimiento intelectual abriendo puertas para la formación del presente y se puedan desenvolver en cualquier ambiente sin ningún problema, la realidad aumentada es un tema que surgido en los últimos años y ha tenido buenos resultados donde ha evolucionado, ahora es momento de la educación que consiga adaptarla a los diferentes contenidos que se dan en este caso en la asignatura de tecnología, después de haber analizado los resultados se puede llegar a la conclusión según los objetivos específicos.

El primero es diagnosticar las estrategias didácticas empleadas por el docente para el desarrollo de los contenidos de estructuras, donde se pudo observar que el profesor no utiliza muy a manudo estrategias didácticas, pues al trabajar el en aula de tecnología, ya eso le hace ver que está innovando no tomando en cuenta que es necesario la didáctica para que los estudiantes puedan entender los diferentes contenidos que se desarrollan, aunque se cuente con la tecnología es necesaria que se organice y se planifiquen estrategias didácticas y la utilización de recursos tecnológicos.

Seguidamente se presenta, describir las herramientas y recursos que desde la tecnología promueven el empleo de la realidad aumentada, e de resaltar que la institución educativa cuenta con Wi-Fi educativo, así mismo con los equipos que permiten el trabajo con la realidad aumentada, también es de resaltar que los dispositivos que los estudiantes tienen para su uso personal son de alta gama y permiten que por medio de ellos que se logre el trabajo de la misma, las herramientas y recursos 
están, es necesario que exista la capacitación de los docentes y la de los estudiantes para lograr trabajar con ellos cualquier contenido y comprendan la importancia del trabajo con la realidad aumentada desde el punto de vista educativo.

Asimismo se Determinó la factibilidad de aplicación de un material instruccional apoyado en la realidad aumentada para el desarrollo de los contenidos de estructuras del área de tecnología en los estudiantes de séptimo grado de la institución Educativa Colegio "Pablo Correa León", ubicada en el municipio de Cúcuta, por medio de los ítems que se desarrollaron se pudo verificar que existe una factibilidad positiva con el uso de la realidad aumentada en el área de tecnología y sobre todo en contenidos como estructura de datos, algoritmos, y procesos binarios, es interesante que el material instruccional que se piensa diseñar cubra con las expectativas de los estudiantes de séptimo grado.

Finalmente se diseñara un material instruccional para el desarrollo del contenido de estructuras mediante la aplicación de la realidad aumentada en el área de tecnología en la institución objeto de estudio, después de haber revisado los resultados se procederá a diseñar el material de una manera didáctica para motivar a los jóvenes a trabajar con la realidad aumentada, y así facilitar el proceso de aprendizaje de los jóvenes en el contenido de estructura, partiendo del área de tecnología.

\section{Recomendaciones del estudio}

Es importante resaltar que por ser temas innovadores, es necesario que los directivos de la institución tomen en cuenta esta investigación y enfoquen la propuesta para darle una posible solución a la problemática existente.

Que la institución sea piloto para para el uso de la realidad aumentada como estrategia didáctica en el proceso de enseñanza.

Brindar la información a otras instituciones educativas con el fin de que ellos puedan observar la importancia de estos temas dentro de la educación y se incentiven implementarla en otras áreas del currículo.

Centrar todo en el estudiante con el fin de mostrarle la importancia de la tecnología, los dispositivos tecnológicos y la realidad aumentada desde el punto de vista educativo.

Que los docentes se mantengan en constante actualización con los diferentes temas tecnológicos que se presentan para poder incorporarlos rápidamente a la educación, ya que es lo que mantiene la atención de los niños, jóvenes y adultos en la actualidad.

\section{Referencias}

Aneca (2004). Libro Blanco del Título de Grado en Ingeniería Informática. Agencia Nacional de Evaluación de la Calidad y Acreditación. Argentina.

Arias, F (2006). El Proyecto de Investigación Introducción a la Metodología Científica. Caracas, Venezuela: Episteme.

Azuma, R. (1997), A Survey of Augmented Reality. Presence: Teleoperators and Virtual Environments 6(4), 355 - 385. Recuperado de: http:// http://www.cs.unc.edu/ azuma/ ARpresence.pdf [Consulta: 2017, marzo 24]

Bimber, O. y Raskar, R. (2005). Realidad aumentada espacial: Real fusión y los mundos virtuales. AK Peters

Cairo, O y Guardati, S. (2006). Estructuras de datos. México: McGraw-Hill.

Castañeda, J. (2006). Métodos de Investigación. México: Editirial Trillas.

De la Torre, Martin, N., Saorín, J., Carbonell, C. y Contero, M. (2011). Entorno de aprendizaje ubicuo con realidad aumentada y tabletas para estimular la comprensión del espacio 
tridimensional. RED. Recuperado de: http:// www.um.es/ead/red/37/DELATORREetAL. pdf. [Consulta: 2017, diciembre 3]

De Luca, S. (2000). El docente y las inteligencias múltiples. Recuperado de: http:// www. rieoei.org/deloslectores/616Luca.PDF. [Consulta: 2017, diciembre 5]

Espinosa, C. (2015). Realidad aumentada y educación: análisis de experiencias prácticas. Píxel-Bit, Revista de Medios y Educación. 46, 187-203.

Gardner, H. (1994). Estructuras de la Mente: La Teoría de las Inteligencias Múltiples. Recuperado de: http://www.slideshare. net/psicologavanessasoto/gardnerhowardestructuras-de-la-mente-9005886. [Consulta: 2017, diciembre 5]

Guerra, M. y Jordán, V. (2010). Políticas públicas de Sociedad de la Información en América Latina: ¿una misma visión? Recuperado de: http://www.cepal.org/ddpe/publicaciones/ xml/1/39181/w314esp.pdf. CEPAL, Santiago de Chile. [Consulta: 2017, Mayo 2]

Hernández y otros (2006). Metodología de la Investigación. México: Mac Graw Hill ediciones Interamericana.

Ministerio de Educación Nacional (2013). Competencias TIC para el desarrollo profesional docente

Ministerio de Educación Nacional. (2006). Estándares Básicos de Competencias.

Ministerio de Educación, Icfes. (2013) Colombia en PISA 2012, Informe nacional de resultados, Resumen ejecutivo. Extraído el 15 de mayo de 2015. Recuperado de: http://www.icfes.gov.co/investigacion/ evaluaciones-internacionales/pisa

Monal, J. (2012). La gestión ambiental apoyada por realidad aumentada, para el desarrollo del pensamiento social en estudiantes del grado noveno. Trabajo de grado de maestría no publicado. Universidad Tecnológica De Pereira

Novoa, P (2013). Dispositivo interactivo con realidad aumentada para el desarrollo de competencias musicales. Trabajo de grado de pregrado no publicado. Universidad Católica de Pereira. Extraído el 3 de mayo de 2015. Recuperado de: http:// ribuc.ucp.edu.co:8080/jspui/bitstream/ handle/10785/1688/CDMDI334. pdf? sequence $=1$

Reinoso, R. (2014). Introducción a la Realidad Aumentada. Recuperado de: http://crol. crfptic.es/archivos/3711.PDF. [Consulta: 2017, mayo 13]

Ruiz, C. (2002). Instrumentos de Medición en Ciencias Sociales. FEDUPEL. Caracas.

Ruiz, D. (2011). Realidad Aumentada, educación y museos. Revista Icono 14. (2), 212-226. (ISSN 1697-8293)

Sabino, C. (1992). El Proceso de Investigación. Caracas: Panapo.

Saurith, A (2014). Competencias TIC para el desarrollo profesional docente: una oportunidad para innovar. Revista Iberoamericana de Educación. Ruta maestra. Edición 6. Santillana. Colombia. Extraído el 3 de marzo de 2017. Recuperado de: www. santillana.com.co/rutamaestra/edicion-6/ pdf/11.pdf

Tamayo, C. (2008). Métodos de Investigación. México: Trillas.

Universidad Pedagógica Experimental Libertador UPEL (2010). Manual de Presentación de Trabajos de Grado de Especialización, Maestría y tesis Doctorales. FEDUPEL. Caracas. 